\section{Singlemode InAs quantum dot photonic crystal VCSELs}

H.P.D. Yang, Y.H. Chang, F.I. Lai, H.C. Yu, Y.J. Hsu, G. Lin, R.S. Hsiao, H.C. Kuo, S.C. Wang and J.Y. Chi

\begin{abstract}
An InAs quantum dot photonic crystal vertical-cavity surface-emitting laser (QD PhC-VCSEL) for fibre-optic applications is first demonstrated. Single fundamental mode $\mathrm{CW}$ output power of $0.2 \mathrm{~mW}$ has been achieved in the $1300 \mathrm{~nm}$ range, with a threshold current of $4.75 \mathrm{~mA}$. Side-mode suppression ratio larger than $40 \mathrm{~dB}$ has been observed over the entire thermally limited operation range.
\end{abstract}

Introduction: Vertical-cavity surface-emitting lasers (VCSELs) have attracted much attention in recent years. Singlemode VCSELs are needed for a number of applications, including high-speed laser printing, optical storage and long-wavelength telecommunications. For oxide-confined VCSELs, the current-confined aperture must be less than $3 \mu \mathrm{m}$ in diameter to ensure stable singlemode operation [1]. However, the large resistance inherited from the small aperture limits the modulation bandwidth and degrades the high-speed performance. The lifetime of the oxide VCSEL also decreases proportionally as the diameter of the oxide aperture shrinks, even when the device is operated at a reduced current [1]. Methods reported to solve the problem include the increase of higher-order mode loss by surfacerelief etching [2] and hybrid oxide-implanted VCSELs [3, 4]. Recently, a two-dimensional photonic crystal (2-D PhC) structure formed on the VCSEL surface has been investigated as a control method of lateral mode. Singlemode output was realised from larger aperture photonic crystal VCSELs (PhC-VCSELs) [5, 6]. However, those PhC-VCSELs exhibit relatively high threshold currents $\left(I_{\mathrm{th}}\right)$ owing to large oxide-confined apertures. For long-wavelength applications, InGaNAs [7, 8] and InAs-InGaAs quantum dot (QD) VCSELs [9] achieved laser emission at $1300 \mathrm{~nm}$. Singlemode operation of the QD VCSEL with PhC is yet to be realised. In this Letter, we report our results on the QD PhC-VCSELs in the $1300 \mathrm{~nm}$ range. Single-lateral-mode operation with very high side-mode suppression ratio (SMSR) is demonstrated for the first time.

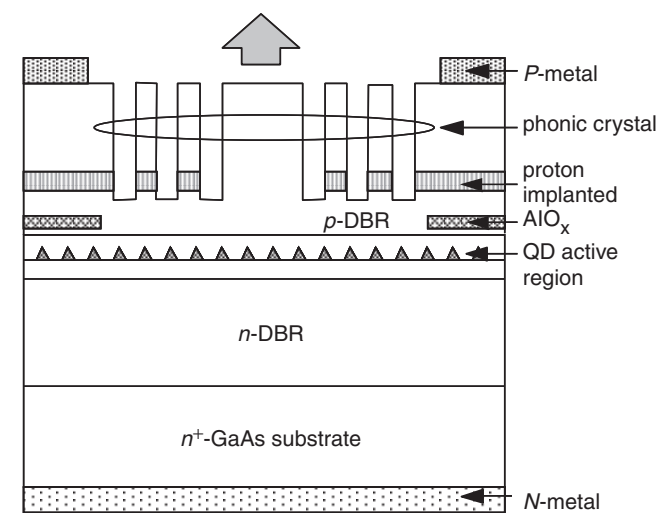

Fig. 1 Schematic of $Q D P h C-V C S E L$

Hole etching depth of $\mathrm{PhC}$ is 18 pairs out of the 23-pair top DBR having been etched off. The proton implantation position is 10 pairs of DBR layers above active region

Experiment: The epitaxial layers of the QD PhC-VCSEL wafers were grown on $n^{+}$-GaAs substrates by molecular beam epitaxy (MBE). The bottom distributed Bragg reflector (DBR) consists of a 33.5-pair $n$-type (Si-doped) quarter-wave stack $(\lambda / 4)$ of $\mathrm{Al}_{0.9} \mathrm{Ga}_{0.1} \mathrm{As} / \mathrm{GaAs}$. The top DBR consists of a 23-period $p$-type (carbon-doped) $\mathrm{Al}_{0.9} \mathrm{Ga}_{0.1} \mathrm{As} / \mathrm{GaAs}$ quarter-wave stack. Above that, is a heavily doped $p$-type GaAs contact layer. The active region contains 17 undoped InAs-InGaAs QD layers, separated by GaAs barrier layers. Each of the InAs-InGaAs QD layers consists of 2.5 monolayer-thick InAs pyramidal islands and a 5 nm-thick $\operatorname{In}_{0.15} \mathrm{Ga}_{0.85}$ As quantum well overgrowth layer. The current confinement of the device was achieved by combining selective oxidation with proton $\left(\mathrm{H}^{+}\right)$-implantation. First, mesas with diameters varied from 44 to $68 \mu \mathrm{m}$ were defined by reactive ion etch (RIE). The AlAs layer within the $\mathrm{Al}_{0.9} \mathrm{Ga}_{0.1} \mathrm{As}$ confinement layers was selectively oxidised to $\mathrm{AlO}_{\mathrm{x}}$. The oxidation depth was about $12 \mu \mathrm{m}$ towards the centre from the mesa edge so that the resulting oxide aperture varied from 20 to $44 \mu \mathrm{m}$ in diameter. The oxide aperture was introduced to reduce the lateral optical loss and the leakage current. The p-contact ring with an inner diameter $2 \mu \mathrm{m}$ larger than the oxide aperture was formed on the top of the p-contact layer. The $n$-contact was formed at the bottom of the $n^{+}$-GaAs substrate. After that, triangular lattice patterns of photonic crystal with a singlepoint defect in the centre were defined within the p-contact ring using photolithography and etched through the $p$-type DBR using RIE. The lateral index around a single defect can be controlled by the hole diameter $(\alpha)$-to-lattice constant $(\Lambda)$ ratio and etching depth [5]. This ratio $(\alpha / \Lambda)$ is 0.5 ; the lattice constant $\Lambda$ is $5 \mu \mathrm{m}$ in the PhC-VCSEL and the etching depth of the holes is about 18 pairs thick into the 23-pair top DBR layer. To ensure better current confinement of the device, proton implantation was carried out with a diameter of $12 \mu \mathrm{m}$, followed by an annealing at $430^{\circ} \mathrm{C}$ under $\mathrm{N}_{2}$ ambient. The implantation energy was $240 \mathrm{keV}$, with a dose of $6 \times 10^{14} \mathrm{~cm}^{-2}$, to form an insulating region laying $10 \mathrm{DBR}$ pairs above the active region. Higher implantation energy may introduce more defects in the lateral direction. The device structure is shown in Fig. 1. By using two types of apertures in this device, we decouple the effects of the current confinement from the optical confinement. The $\mathrm{H}^{+}$implant aperture $(12 \mu \mathrm{m})$ and the $\mathrm{AlO}_{\mathrm{x}}$ layer are used to confine the current flow, while the single-point defect ( $\geq 12 \mu \mathrm{m}$ in diameter) photonic crystal is used to confine the optical mode. To clarify the effect of the photonic crystal index-guiding layer, a VCSEL with $\mathrm{H}^{+}$implant aperture (12 $\mu \mathrm{m}$ in diameter without $\mathrm{PhC}$ ) was also fabricated for comparison.

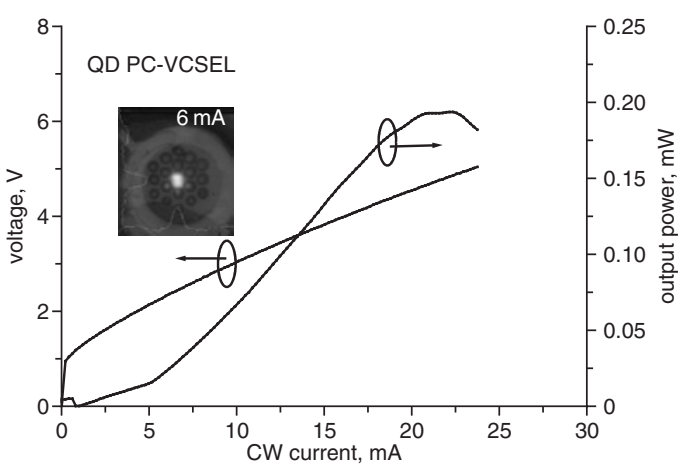

Fig. $2 C W L-I-V$ characteristics and near-field image (inset) of PhCVCSEL (ratio $(\alpha / \Lambda)$ is 0.5 and lattice constant $\Lambda$ is $5 \mu \mathrm{m}$ )
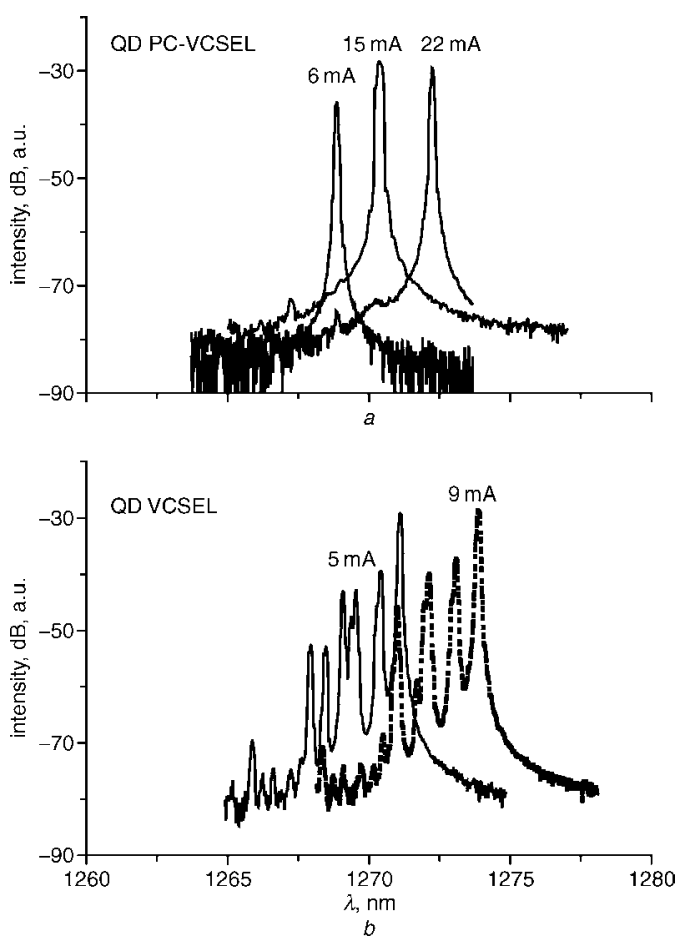

Fig. 3 Spectra of $Q D$

$a$ PhC-VCSEL

$b$ VCSEL without $\mathrm{PhC}$ holes 
Results and discussion: Fig. 2 shows the $\mathrm{CW}$ light-current-voltage $(L-I-V)$ output and near-field image operated at $6 \mathrm{~mA}$ (inset) of the PhC-VCSEL. The VCSEL emits $0.2 \mathrm{~mW}$ peak power and exhibits single modes throughout the current range of operation. The threshold current $\left(I_{\mathrm{th}}\right)$ of the PhC-VCSEL is $4.75 \mathrm{~mA}$. The $I-V$ characteristics exhibit higher series resistance for the PhC-VCSEL, which should be mainly due to proton implantation through the p-ohmic contact of the device and blocking of the current flow in the region by photonic crystal holes. The differential series resistance is $170 \Omega$ at $12 \mathrm{~mA}$. The output power could be improved by reducing the series resistance of the PhC-VCSEL. Lasing spectra of the PhC-VCSEL is shown in Fig. $3 a$, confirming singlemode operation within the overall operation current. The peak lasing wavelengths are 1268 and $1272 \mathrm{~nm}$ at 6 and $22 \mathrm{~mA}$, respectively. The PhC-VCSEL exhibits an SMSR $>40 \mathrm{~dB}$ throughout the current range. For comparison, a lasing spectra of a QD VCSEL without photonic crystal holes shows multiple mode operation as the driving current increased above $5 \mathrm{~mA}$ (Fig. $3 b$ ). The QD VCSEL showed multiple transverse mode characteristics over a broader wavelength span.

Conclusion: We report a singlemode QD PhC-VCSEL with SMSR $>40 \mathrm{~dB}$ throughout the operation current range. The present results indicate that a VCSEL using a combined oxide layer with proton implantation for current confinement and photonic crystal for optical confinement is a promising approach to achieve singlemode operation of VCSELs.

Acknowledgments: The authors wish to thank A. R. Kovsh and S. S. Mikhrin of NL Nanosemiconductor GmbH, Germany, for providing the QD-VCSEL epitaxial wafers. This work was supported by Nanophotonics Project, MOEA, Taiwan.

(C) IEE 2005

Electronics Letters online no: 20052518

26 July 2005

doi: 10.1049/el:20052518

H.P.D. Yang, H.C. Yu, G. Lin, R.S. Hsiao and J.Y. Chi (Nanophotonic Center, Industrial Technology Research Institute, Chutung 310, Hsinchu, Taiwan)

E-mail: hpyang@itri.org.tw
Y.H. Chang, F.I. Lai, Y.J. Hsu, H.C. Kuo and S.C. Wang (Institute of Electro-Optical Engineering, National Chiao Tung University, 1001 Ta Hsueh Road, Hsinchu, Taiwan 30050)

H.C. Yu: Also with the Institute of Microelectronics, National Cheng Kung University, Tainan 701, Taiwan

\section{References}

1 Hawkins, B.M., Hawthorne III, R.A., Guenter, J.K., Tatum, J.A., and Biard, J.R.: 'Reliability of various size oxide aperture VCSELs'. Proc. 52nd Electronic Components and Technology Conf., 2002, pp. $540-550$

2 Haglund, A., Gustavsson, J.S., Vukusic, J., Modh, P., and Larsson, A.: 'Single fundamental mode output power exceeding $6 \mathrm{~mW}$ from VCSELs with a shallow surface relief', IEEE Photonics Technol. Lett., 2004, 16, (2), pp. 368-370

3 Hsueh, T.-H., Kuo, H.-C., Lai, F.-I., Laih, L.-H., and Wang, S.C.: 'Highspeed characteristics of large-area single-transverse-mode vertical-cavity surface-emitting lasers', Electron. Lett., 2003, 39, (21), pp. 1519-1521

4 Young, E.W., Choquette, K.D., Chuang, S.L., Geib, K.M., Fischer, A.J., and Allerman, A.A.: 'Single-transverse-mode vertical-cavity lasers under continuous and pulsed operation', IEEE Photonics Technol. Lett., 2001, 13, (9), pp. 927-929

5 Yokouchi, N., Danner, A.J., and Choquette, K.D.: 'Etching depth dependence of the effective refractive index in two-dimensional photonic-crystal-patterned vertical-cavity surface-emitting laser structures', Appl. Phys. Lett., 2003, 82, (9), pp. 1344-1346

6 Berkedal, D., Gregersen, N., Bischoff, S., Madsen, M., Romsted, F., and Oestergarrd, J.: 'Large-area single-mode photonic bandgap VCSELs'. Proc. Optical Fiber Communication Conf., Atlanta, GA, USA, pp. 83-85

7 Vukusic, J., Modh, P., Larsson, A., Hammar, M., Mogg, S., Christiansson, U., Oscarsson, V., Ödling, E., Malmquist, J., Ghisoni, M., Gong, P., Griffiths, E., and Joel, A.: 'MOVPE-grown GaInNAs VCSELs at $1.3 \mu \mathrm{m}$ with conventional mirror design approach', Electron. Lett., 2003, 39, (8), pp. 662-664

8 Wang, J.S., Hsiao, R.S., Lin, G., Lin, K.F., Liu, H.Y., Lai, C.M., Wei, L., Liang, C.Y., Chi, J.Y., Kovsh, A.R., Maleev, N.A., Livshits, D., Chen, J.F., Yu, H.C., and Ustinov, V.M.: 'Molecular-beam-epitaxy growth of highquality InGaNAs/GaAs quantum well lasers emitting at $1.3 \mu \mathrm{m}$ ', J. Vac. Sci. Technol., 2004, B22, (6), pp. 2663-2667

9 Lott, J.A., Ledentsov, N.N., Ustinov, V.M., Mallev, N.A., Zhukov, A.E., Kovsh, A.R., Maximov, M.V., Volvovik, B.V., Alferov, Z.H.I., and Bimberg, D.: 'InAs-InGaAs quantum dot VCSELs on GaAs substrates emitting at $1.3 \mu \mathrm{m}$ ', Electron. Lett., 2000, 36, (16), pp. 1384-1385 